\title{
Streptococcal acute pharyngitis
}

\section{Lais Martins Moreira Anjos ${ }^{[1]}$, Mariana Barros Marcondes ${ }^{[1]}$, Mariana Ferreira Lima ${ }^{[1]}$, Alessandro Lia Mondelli ${ }^{[1]}$ and Marina Politi Okoshi[ ${ }^{[1]}$}

[1]. Departamento de Clínica Médica, Faculdade de Medicina de Botucatu, Universidade Estadual Paulista, Botucatu, SP.

\begin{abstract}
Acute pharyngitis/tonsillitis, which is characterized by inflammation of the posterior pharynx and tonsils, is a common disease. Several viruses and bacteria can cause acute pharyngitis; however, Streptococcus pyogenes (also known as Lancefield group A $\beta$-hemolytic streptococci) is the only agent that requires an etiologic diagnosis and specific treatment. S. pyogenes is of major clinical importance because it can trigger post-infection systemic complications, acute rheumatic fever, and post-streptococcal glomerulonephritis. Symptom onset in streptococcal infection is usually abrupt and includes intense sore throat, fever, chills, malaise, headache, tender enlarged anterior cervical lymph nodes, and pharyngeal or tonsillar exudate. Cough, coryza, conjunctivitis, and diarrhea are uncommon, and their presence suggests a viral cause. A diagnosis of pharyngitis is supported by the patient's history and by the physical examination. Throat culture is the gold standard for diagnosing streptococcus pharyngitis. However, it has been underused in public health services because of its low availability and because of the 1- to 2-day delay in obtaining results. Rapid antigen detection tests have been used to detect $S$. pyogenes directly from throat swabs within minutes. Clinical scoring systems have been developed to predict the risk of $S$. pyogenes infection. The most commonly used scoring system is the modified Centor score. Acute S. pyogenes pharyngitis is often a self-limiting disease. Penicillins are the first-choice treatment. For patients with penicillin allergy, cephalosporins can be an acceptable alternative, although primary hypersensitivity to cephalosporins can occur. Another drug option is the macrolides. Future perspectives to prevent streptococcal pharyngitis and post-infection systemic complications include the development of an anti-Streptococcus pyogenes vaccine.
\end{abstract}

Keywords: Acute rheumatic fever. Antigen. Penicillin. Streptococcal infection. Therapeutics.

\section{INTRODUCTION}

Acute pharyngitis/tonsillitis, characterized by inflammation of posterior pharynx and tonsils, is a common condition observed in outpatients seeking healthcare provision. Its main symptoms are sore throat and fever. Most cases of acute pharyngitis present a benign course and resolve without antiinfective treatment. Several viruses and bacteria can cause acute pharyngitis; however, Streptococcus pyogenes (also known as Lancefield group A $\beta$-hemolytic streptococci) is the only agent that requires an etiologic diagnosis and specific treatment. $S$. pyogenes is of major clinical importance because it can trigger post-infection systemic complications, acute rheumatic fever, and post-streptococcal glomerulonephritis, which occur 1-3 weeks after the pharynx infection ${ }^{1,2}$.

In the United States of America, there are an estimated 7 million cases of acute pharyngitis diagnosed in children annually ${ }^{2}$. S. pyogenes is responsible for $5 \%$ to $30 \%$ of cases of acute pharyngitis, and it is more frequent in children than in adults ${ }^{1-5}$. It is more common in children between 5 years

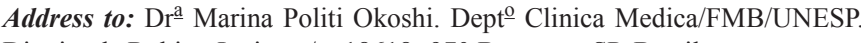
Distrito de Rubião Junior, s/n, 18618- 970 Botucatu, SP, Brasil.

Phone: 5514 3880-1171; Fax: 5514 3882-2238

e-mail:mpoliti@fmb.unesp.br

Received 19 December 2013

Accepted 29 May 2014
}

and 15 years ${ }^{1,6}$. In Brazil, it is difficult to evaluate the incidence of streptococcal infections; however, the Brazilian Institute of Geography and Statistics (Rio de Janeiro, Brazil) estimates that 10 million cases of streptococcal infection occur annually ${ }^{5}$. In this review, we present the cause and clinical manifestations of acute pharyngitis, and the diagnosis and treatment of streptococcal pharyngitis.

\section{ETIOLOGY AND CLINICAL MANIFESTATIONS}

Viruses cause approximately $75 \%$ of pharyngitis cases. The following viruses have been associated with acute pharyngitis: rhinovirus, coronavirus, adenovirus, influenza virus, parainfluenza virus, coxsackievirus, herpes simplex virus, Epstein-Barr virus, cytomegalovirus, and human immunodeficiency virus. In addition to group A streptococci, several strains of bacteria can cause acute pharyngitis such as group C streptococci and group G streptococci, Fusobacterium necrophorum, Arcanobacterium haemolyticum, Neisseria gonorrhoeae, Treponema pallidum, Francisella tularensis, Corynebacterium diphtheriae, Yersinia enterocolitica, Yersinia pestis, Mycoplasma pneumoniae, Chlamydophila psittaci, and mixed anaerobes ${ }^{1}$.

Common manifestations of acute pharyngitis are fever and sore throat with or without tonsillar erythema, swelling, exudate, or ulcerations. In streptococcal infections, symptom onset is usually abrupt and includes intense sore throat, fever, chills, malaise, headache, tender and enlarged anterior cervical lymph nodes, and pharyngeal or tonsillar exudate ${ }^{1,2}$. Palatal petechiae 
and scarlatiniform rash are highly specific, but rarely present ${ }^{6}$. Cough, coryza, conjunctivitis, and diarrhea are uncommon in streptococcal infection, and their presence suggests a viral etiology.

\section{DIAGNOSIS}

A diagnosis of pharyngitis is supported by the patient's medical history and by the physical examination. Previous exposure to streptococcus or viral agents should be investigated. However, infection caused by many other agents can be clinically indistinguishable from streptococcal pharyngitis ${ }^{1}$.

The etiologic diagnosis is based on laboratory tests. Throat culture is the gold standard for diagnosing pharyngitis caused by streptococcus and it has a sensitivity ranging between $90 \%$ and $95 \%$. Samples should be obtained by vigorously swabbing the tonsils and the posterior pharyn $\mathrm{x}^{2}$. If the result is negative, most patients should not be administered antibiotic therapy. However, when the result is positive, it does not eliminate the possibility of chronic colonization'. In Brazil, throat culture has been underused in public health services because of its low availability and because of the long (i.e., 1-2 day) period needed to obtain results.

Rapid antigen detection tests have been used to detect $S$. pyogenes directly from throat swabs within minutes ${ }^{1,2,7}$. These tests have a high specificity (89.7\%-99.0\%) and variable sensitivity (55.0\%-99.0\%), depending on the assay used and the probability of streptococcal infection ${ }^{1-3,6,8}$. When results are positive, the rapid tests guide treatment and dispense with the need for a throat culture. However, if results are negative, clinicians should decide between performing a throat culture or ruling out streptococcal infection, based on the clinical evidence of S. pyogenes and their health service guidelines ${ }^{3}$. The recent European guidelines state that a negative rapid test is sufficient to rule out streptococcal infection and discharge the patient ${ }^{8}$. In children, a negative rapid test can be confirmed by throat culture if there is a high clinical and epidemiologic suspicion of streptococcal pharyngitis ${ }^{6}$. The test is quick to perform; however, some clinicians use rapid tests less frequently than recommended ${ }^{9}$. A recent French study shows that the three primary barriers against using rapid tests are: I) the time required to perform the test; II) patient/parent expectations regarding antibiotics; and III) the perception that the clinical examination is sufficient for prescribing an antibiotic ${ }^{10}$. Furthermore, rapid tests are not available in all public health services, particularly in underdeveloped countries.

Anti-streptococcal antibody titers are not useful in diagnosing acute infection because they increase later. For example, the level of one of the most commonly used antistreptococcal antibody tested, anti-streptolysin $\mathrm{O}$, starts to increase from 7 to 14 days and peaks 3-6 weeks after the beginning of the infection ${ }^{2}$. The C-reactive protein level and the leukocyte count are not recommended for diagnosing streptococcal pharyngitis ${ }^{6}$, although these tests can be useful in monitoring infection progress and treatment outcome.

Although etiologic diagnosis of streptococcal pharyngitis depends on the laboratory tests, clinical scoring systems have been developed to predict the risk of $S$. pyogenes infection ${ }^{11,12}$.
The most commonly used scoring system is the Centor score, which was first proposed in 1981 and modified in 2004 to include age in the risk classification (Table 1) ) $^{2,13,14}$. The authors clinically estimated the risk of streptococcal pharyngitis by using fever, cough, tender anterior cervical adenopathy, tonsillar swelling or exudate, and patient age data (Table 2). The Centor score was recently validated in an analysis of data collected from 206,870 patients aged 3 years or more with a painful throat ${ }^{15}$. Several guidelines recommend the score as a triage method ${ }^{6}$.

Despite the convenience of the Centor score for risk classification of streptococcal pharyngitis, its clinical use is controversial $^{16}$. For patients with a score of 1 point or less or a score of 4 points or greater, there seems to be consensus among several guidelines ${ }^{1,6,8,17}$. Patients scoring 1 point or less with a streptococcal infection risk of up to $10 \%$ should be released without the need for laboratorial investigation and antibiotic therapy. The exception to this rule is a patient with documented exposure to $S$. pyogenes in the preceding 2 weeks or with a history of acute rheumatic fever or cardiac rheumatic disease ${ }^{1}$. Patients scoring 4 points or more should receive antibiotic therapy following an initial clinical evaluation ${ }^{2}$.

However, for patients with Centor scores of 2 points or 3 points, there is great controversy surrounding the laboratorial investigation and treatment that follows, even in developed countries. In the United States and several European countries where the incidence of rheumatic fever is low, physicians should perform a laboratory investigation and prescribe antibiotics only after obtaining positive throat culture or rapid test results ${ }^{10}$. In contrast, acute pharyngitis in other European countries is considered a benign self-limiting disease and microbiological tests are not routinely recommended; antibiotic treatment is reserved for well-selected patients ${ }^{6}$.

In Brazil and in underdeveloped countries, the incidence of rheumatic fever remains elevated. An estimated 282,000 new cases of rheumatic heart disease occur each year worldwide; most of these cases are in underdeveloped countries ${ }^{18}$. In addition, public health services in Brazil experience difficulty

TABLE 1 - The modified Centor score to use for children and adults with a sore throat to estimate probability of Streptococcus pyogenes infection*.

\begin{tabular}{lc}
\hline Criteria & Points \\
\hline Fever (temperature $>38^{\circ} \mathrm{C}$ ) & +1 \\
Absence of cough & +1 \\
Swollen and tender anterior cervical nodes & +1 \\
Tonsillar swelling or exudates & +1 \\
\hline Age (years) & +1 \\
$3-14$ & 0 \\
$15-44$ & -1 \\
$\geq 45$ & \\
\hline
\end{tabular}

*Information is adapted from McIsaac et al. ${ }^{13}$ and Low ${ }^{2}$. 
TABLE 2 - Risk for streptococcal infection*.

\begin{tabular}{lcc}
\hline Score (points) & Risk $(\%)$ & Recommendation \\
\hline$\leq 0$ & $1-2.5$ & No laboratorial investigation or ATB prescription \\
1 & $5-10$ & No laboratorial investigation or ATB prescription \\
2 & $11-17$ & Controversial \\
3 & $28-35$ & Controversial \\
$\geq 4$ & $51-53$ & ATB prescription \\
\hline
\end{tabular}

ATB: antibiotic therapy; *Information is adapted from McIsaac et al. ${ }^{13}$ and Low $^{2}$.

in performing culture tests and rapid tests. Therefore, we recommend that, if a laboratory investigation is unavailable and depending on clinical judgment, patients with a Centor score of 2 points or greater should be treated with antibiotics. However, because adults with acute pharyngitis have a low incidence of $S$. pyogenes infection and acute rheumatic fever, using the Centor score without laboratory confirmation could result in a large number of adults with non-streptococcal pharyngitis being treated with antibiotics ${ }^{17}$.

\section{TREATMENT}

There are recommendation discrepancies between the European and North American guidelines for acute pharyngitis treatment, both of which emphasize the importance of reducing overall antibiotic use to limit antibiotic resistance ${ }^{6,8}$. However, many physicians still prescribe antibiotics in patients with pharyngitis without evidence of $S$. pyogenes infection ${ }^{3,6,9,19,20}$. Because most acute pharyngitis cases are caused by viruses, antibiotic treatment is completely useless. A recent report showed that antibiotics were prescribed for up to $73 \%$ of adults with acute pharyngitis ${ }^{3}$. In fact, acute pharyngitis is a major cause of inappropriate antibiotic use in clinical practice ${ }^{3}$.

Acute $S$. pyogenes pharyngitis is often a self-limiting disease. Fever usually resolves within 3-5 days and sore throat resolves in 1 week. ${ }^{1}$ Antibiotics help to reduce the severity and duration of symptoms, limit disease spread, and prevent suppuration (e.g., peritonsillar or retropharyngeal abscess, cervical lymphadenitis, otitis media, and mastoiditis) and noninfectious complications such as acute rheumatic fever ${ }^{1,3,6,21}$. There is some doubt whether post-streptococcal glomerulonephritis can be prevented by antibiotic treatment in streptococcal pharyngitis ${ }^{1}$. Antibiotics are less efficient in improving symptoms when started 2 days after disease initiation ${ }^{3}$. However, even when started 1-2 days after symptoms have begun, they are equally effective in preventing rheumatic fever ${ }^{5,17}$. Patients are considered noncontagious or only minimally contagious 24 hours after starting antibiotic treatment ${ }^{16,22}$; therefore, children should return to school $24 \mathrm{~h}$ after starting antibiotic therapy.

Narrow-spectrum penicillins are the first choice for treatment because of the rarity of documented resistance to penicillin by group A streptococcus during pharyngitis treatment and because of their low $\operatorname{cost}^{2,6,23}$. A recent literature review failed to show differences between antibiotics in treating streptococcal pharyngitis ${ }^{23}$.
The recommended oral formulation is penicillin V. For complete agent eradication, it is important to emphasize that oral penicillin should be taken for 10 days, even if symptoms subside within a few days ${ }^{5,6}$. It is difficult for patients to maintain treatment for 10 days because of the drug's poor palatability, the need to take it several times a day, and the rapid symptom resolution. Because amoxicillin is reportedly equally effective and has a better palatability, it is a suitable option for children ${ }^{6}$. The Brazilian guidelines for rheumatic fever prevention recommend a single dose of intramuscular benzathine penicillin as the first choice for treating streptococcal pharyngitis. This treatment has a low incidence of side effects and a high rate of adherence and streptococcus eradication ${ }^{5}$. Table 3 shows antibiotic dosing regimens ${ }^{5,6}$. In patients with penicillin allergy, cephalosporins can be an acceptable alternative, although a primary hypersensitivity to cephalosporins can occur 6 . The macrolides are another option (Table 3). S. pyogenes resistance to erythromycin has increased with increased macrolide use ${ }^{24}$.

A recent literature review shows that the administration of new generation antibiotics such as azithromycin for 3-6 days has comparable efficacy as the standard 10-day course of oral penicillin ${ }^{25}$. However, the authors of that review state that this result must be interpreted with caution in areas with a high incidence of rheumatic heart disease.

Anti-inflammatory agents such as ibuprofen, ketoprofen, and diclofenac, or analgesic agents such as paracetamol can reduce severe symptoms and high fever ${ }^{8}$. Systemic corticosteroids should not be routinely prescribed in acute pharyngitis ${ }^{8,26}$. They can however be considered in adult patients who have more severe presentations (e.g., a score of 3-4 points in the Centor criteria) ${ }^{8}$.

In a recurrence of pharyngitis, a throat swab culture or a rapid antigen detection test should be performed. When test results are positive, patients should be treated again. In these cases, it is also recommended to detect and treat healthy carriers among people from the same residence ${ }^{1}$.

\section{FUTURE PERSPECTIVES}

Future perspectives on preventing streptococcal pharyngitis, and more importantly systemic post-infection complications include the development of an anti-Streptococcus pyogenes vaccine $^{27}$. Several decades ago, efforts to produce a vaccine against $S$. pyogenes began, and different models have been proposed ${ }^{27}$. There are currently several vaccine models, most in 
TABLE 3 - Treatment for group A Streptococcus pharyngitis.

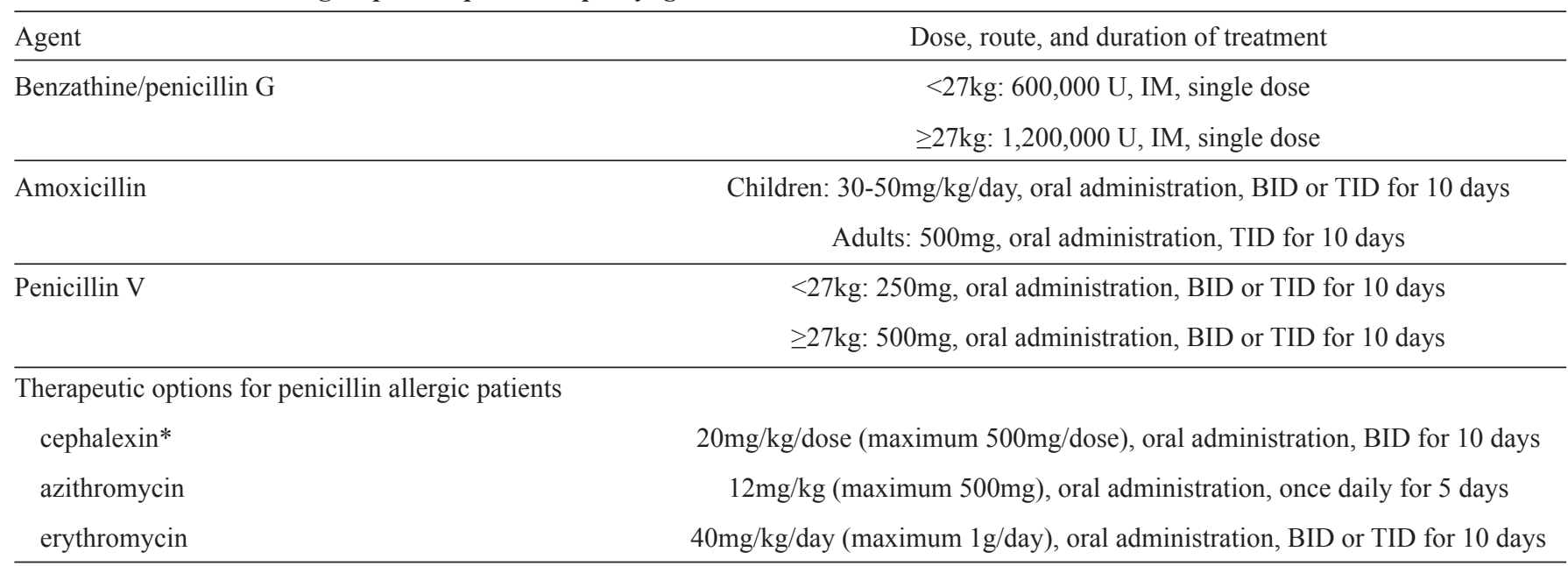

U: units; IM: intramuscular; BID: twice a day; TID: three times a day. *Patients with immediate or type I hypersensitivity to penicillin should not be treated with a cephalosporin ${ }^{5,6}$.

preclinical studies. ${ }^{5}$ Recent experimental studies have shown that a 55-residue peptide, called StreptInCor, can be an effective and safe vaccine for preventing $S$. pyogenes infection ${ }^{28,29}$.

In conclusion, acute pharyngitis/tonsillitis is a common health issue caused by several viruses and bacteria. S. pyogenes is the only agent that requires an etiologic diagnosis and specific treatment because of its potential to trigger systemic postinfection complications. A diagnosis of pharyngitis is supported by medical history and physical examination. The clinical Centor score has been used to predict the risk of $S$. pyogenes infection. Throat culture is the gold standard for diagnosing streptococcal pharyngitis. Rapid antigen detection tests have been used to detect $S$. pyogenes directly from throat swabs within minutes. Narrow-spectrum penicillins are still the first choice for treating pharyngitis and preventing acute rheumatic fever.

\section{ACKNOWLEDGMENTS}

The authors thank Colin Edward Knaggs for English editing.

\section{CONFLICT OF INTEREST}

The authors declare that there is no conflict of interest.

\section{FINANCIAL SUPPORT}

This work was supported by Conselho Nacional de Desenvolvimento Científico e Tecnológico (CNPq, Brasília, Brazil, Proc. number 306857/2012-0).

\section{REFERENCES}

1. Wessels MR. Streptococcal pharyngitis. N Engl J Med 2011; 364: 648-655.

2. Low DE. Nonpneumococcal streptococcal infections, rheumatic fever. In: Goldman L, Schafer AI, editors. Goldman's Cecil Medicine. 24 $4^{\text {th }}$ edition. Philadelphia: Elsevier Saunders. Volume 2, 2012. p. 1823-1829.

3. Nakhoul GN, Hickner J. Management of adults with acute streptococcal pharyngitis: minimal value for backup strep testing and overuse of antibiotics. J Gen Intern Med 2013; 28:830-834.

4. Anderson NW, Buchan BW, Mayne D, Mortensen JE, Mackey TL, Ledeboer NA. Multicenter clinical evaluation of the illumigene group A Streptococcus DNA amplification assay for detection of group A Streptococcus from pharyngeal swabs. J Clin Microbiol 2013; 51: 1474-1477.

5. Barbosa PJB, Müller RE. Sociedade Brasileira de Cardiologia, Sociedade Brasileira de Pediatria e Sociedade Brasileira de Reumatologia. Diretrizes Brasileiras para Diagnóstico, Tratamento e Prevenção da Febre Reumática. Arq Bras Cardiol 2009; 93 (supl 4):1-18.

6. Chiappini E, Regoli M, Bonsignori F, Sollai S, Parretti A, Galli L, et al. Analysis of different recommendations from international guidelines for the management of acute pharyngitis in adults and children. Clin Ther 2011; 33:48-58.

7. Cardoso DM, Gilio AE, Hsin SH, Machado BM, Paulis M, Lotufo JPB, et al. Impact of the rapid antigen detection test in diagnosis and treatment of acute pharyngotonsillitis in a pediatric emergency room. Rev Paul Pediatr 2013; 31:4-9.

8. Pelucchi C, Grigoryan L, Galeone C, Esposito S, Huovinen P, Little P, et al. Guideline for the management of acute sore throat. ESCMID Sore Throat Guideline Group. Clin Microbiol Infect 2012; 18 (supl 1):1-28.

9. Linder JA, Bates DW, Lee GM, Finkelstein JA. Antibiotic treatment of children with sore throat. JAMA 2005; 294:2315-2322.

10. Pulcini C, Pauvif L, Paraponaris A, Verger P, Ventelou B. Perceptions and attitudes of French general practitioners towards rapid antigen diagnostic tests in acute pharyngitis using a randomized case vignette study. J Antimicrob Chemother 2012; 67:1540-1546. 
11. Irlam J, Mayosi BM, Engel M, Gaziano TA. Primary prevention of acute rheumatic fever and rheumatic heart disease with penicillin in South African children with pharyngitis: a cost-effectiveness analysis. Circ Cardiovasc Qual Outcomes 2013; 6:343-351.

12. Orofino DH, Passos SR, Andrade CA, Nigri D, Santos Werneck L, Oliveira RC, et al. Accuracy and interobserver variation of three clinical decision rules for the diagnosis of streptococcal pharyngitis. Pediatr Infect Dis J 2013; 32:686-687.

13. Centor RM, Witherspoon JM, Dalton HP, Brody CE, Link K. The diagnosis of strep throat in adults in the emergency room. Med Decis Making 1981; 1:239-246.

14. McIsaac WJ, Kellner JD, Aufricht P, Vanjaka A, Low DE. Empirical validation of guidelines for the management of pharyngitis in children and adults. JAMA 2004; 291:1587-1595.

15. Fine AM, Nizet V, Mandl KD. Large-scale validation of the Centor and McIsaac scores to predict group A streptococcal pharyngitis. Arch Intern Med 2012; 172:847-852.

16. Roggen I, van Berlaer G, Gordts F, Pierard D, Hubloue I. Centor criteria in children in a paediatric emergency department: for what it is worth. BMJ Open 2013; 3:e002712.

17. Gerber MA, Baltimore RS, Eaton CB, Gewitz M, Rowley AH, Shulman ST, et al. Prevention of rheumatic fever and diagnosis and treatment of acute Streptococcal pharyngitis: a scientific statement from the American Heart Association Rheumatic Fever, Endocarditis, and Kawasaki Disease Committee of the Council on Cardiovascular Disease in the Young, the Interdisciplinary Council on Functional Genomics and Translational Biology, and the Interdisciplinary Council on Quality of Care and Outcomes Research: endorsed by the American Academy of Pediatrics. Circulation 2009; 119:1541-1551.

18. Carapetis JR, Steer AC, Mulholland EK, Weber M. The global burden of group A streptococcal diseases. Lancet Infect Dis 2005; 5:685-694.

19. Urkin J, Allenbogen M, Friger M, Vinker S, Reuveni H, Elahayani A. Acute pharyngitis: low adherence to guidelines highlights need for greater flexibility in managing paediatric cases. Acta Paediatr 2013; 102:1075-1080.
20. Crocker A, Alweis R, Scheirer J, Schamel S, Wasser T, Levingood K. Factors affecting adherence to evidence-based guidelines in the treatment of URI, sinusitis, and pharyngitis. J Community Hosp Intern Med Perspect 2013; 3. doi: 10.3402/jchimp.v3i2.20744.

21. Del Mar CB, Glasziou PP, Spinks AB. Antibiotics for sore throat. Cochrane Database Syst Rev 2006; 4:CD000023.

22. Martin JM, Green M. Group A streptococcus. Semin Pediatr Infect Dis 2006; 17:140-148.

23. van Driel ML, De Sutter AI, Keber N, Habraken H, Christiaens T. Different antibiotic treatments for group A streptococcal pharyngitis. Cochrane Database Syst Rev 2013; 4:CD004406.

24. Myers AL, Jackson MA, Selvarangan R, Goering RV, Harrison C. Genetic commonality of macrolide-resistant group A beta hemolytic streptococcus pharyngeal strains. Ann Clin Microbiol Antimicrob 2009; 8:33.

25. Altamimi S, Khalil A, Khalaiwi KA, Milner RA, Pusic MV, Al Othman MA. Short-term late-generation antibiotics versus longer term penicillin for acute streptococcal pharyngitis in children. Cochrane Database Syst Rev 2012; 8:CD004872.

26. Principi N, Bianchini S, Baggi E, Esposito S. No evidence for the effectiveness of systemic corticosteroids in acute pharyngitis, community-acquired pneumonia and acute otitis media. Eur J Clin Microbiol Infect Dis 2013; 32:151-160.

27. Guilherme L, Ferreira FM, Köhler KF, Postol E, Kalil J. A vaccine against Streptococcus pyogenes: the potential to prevent rheumatic fever and rheumatic heart disease. Am J Cardiovasc Drugs 2013; 13:1-4.

28. Postol E, Alencar R, Higa FT, Freschi de Barros S, Demarchi LM, Kalil J, et al. StreptInCor: A candidate vaccine epitope against $S$. pyogenes infections induces protection in outbred mice. PLoS ONE 2013; 8:e60969.

29. De Amicis KM, Freschi de Barros S, Alencar RE, Postól E, Martins CO, Arcuri HA, et al. Analysis of the coverage capacity of the StreptInCor candidate vaccine against Streptococcus pyogenes. Vaccine 2014; 32: $4104-4110$. 